\title{
Inositol monophosphatase 1 (IMPA1) mutation in intellectual disability patients impairs neurogenesis but not gliogenesis
}

\section{T. FIGUEIREDO ${ }^{1,2,3}$, A.P.D. MENDES ${ }^{2}$, D.P. MOREIRA $^{1}$, E. GOULART ${ }^{1}$, D. OLIVEIRA ${ }^{1}$, G.S. KOBAYASHI ${ }^{1}$, S. STERN ${ }^{2,5}$, F. KOK ${ }^{1}$, M.C. MARCHETTO ${ }^{2}$, R. SANTOS ${ }^{2,4, *}$, F.H. GAGE,*, M. $\mathrm{ZATZ}^{1, *}$}

${ }^{1}$ Human Genome and Stem Cell Research Center, Biosciences Institute, University of São Paulo, 106 Rua do Matão, 05508-090, São Paulo, Brazil

${ }^{2}$ Laboratory of Genetics, The Salk Institute for Biological Studies, 10010 North Torrey Pines Road, La Jolla, CA 92037, USA

${ }^{3}$ Faculty of Medicine, Federal University of Alagoas, Tabuleiro do Martins, 57072-900, Maceió, Brazil

${ }^{4}$ University of Paris, Institute of Psychiatry and Neuroscience of Paris (IPNP), INSERM U1266, Laboratory of Dynamics of Neuronal Structure in Health and Disease, 102 rue de la Santé, 75014 Paris, France

${ }^{5}$ Sagol Department of Neurobiology, Faculty of Natural Sciences, University of Haifa, Haifa, 3498838, Israel

${ }^{*}$ These authors contributed equally to this work

Corresponding authors: M. Zatz, mayazatz@usp.br; T. Figueiredo, thalita.figueiredo@famed.ufal.br 


\begin{abstract}
A homozygous mutation in the inositol monophosphatase 1 (IMPA1) gene was recently identified in nine individuals with severe intellectual disability (ID) and disruptive behavior. These individuals belong to the same family from Northeastern Brazil, which has 28 consanguineous marriages and 59 genotyped family members. IMPA1 is responsible for the generation of free inositol from de novo biosynthesis and recycling from inositol polyphosphates and participates in the phosphatidylinositol signaling pathway. To understand the role of IMPA1 deficiency in ID, we generated induced pluripotent stem cells (iPSCs) from patients and neurotypical controls and differentiated these into hippocampal dentate gyrus-like neurons and astrocytes. IMPA1-deficient neuronal progenitor cells (NPCs) revealed substantial deficits in proliferation and neurogenic potential. At low passage NPCs (P1 to P3), we observed cell cycle arrest, apoptosis, progressive change to a glial morphology and reduction in neuronal differentiation. These observations were validated by rescuing the phenotype with myoinositol supplemented media during differentiation of patient derived-iPSCs into neurons and by the reduction of neurogenic potential in control NPCs expressing shIMPA1. Transcriptome analysis showed that NPCs and neurons derived from ID patients have extensive deregulation of gene expression affecting pathways necessary for neurogenesis and upregulation of gliogenic genes. IMPA1 deficiency did not affect cell cycle progression or survival in iPSCs and glial progenitor cells or astrocyte differentiation. Therefore, this study shows that the IMPA1 mutation specifically affects NPC survival and neuronal differentiation.
\end{abstract}




\section{INTRODUCTION}

Intellectual disability (ID) is characterized by significantly below average intellectual functioning, demonstrated by an intelligence quotient below 70, that is associated with adaptive limitations before 18 years of age in at least two of the following areas of skills: communication, self-care, home life, social adaptation, health and safety, the use of community resources or academic functions, leisure and work.[1] Despite its occurrence in different social classes and cultures, ID tends to be more prevalent in lower socioeconomic areas, [2, 3] probably due to environmental factors.[3, 4] ID is one of the most common neurodevelopmental conditions, affecting $1-3 \%$ of the population. $[3,5]$ Understanding the molecular etiology of ID is a major medical and scientific challenge because of its exceptional clinical and genetic heterogeneity. Although environmental factors participate in the etiology of ID, genetic factors seem to make a substantial contribution.[6, 7] Genetic forms of ID are caused by X-chromosome gene defects, copy number variants, autosomal recessive gene mutations and sporadic autosomal dominant de novo mutations.[6-8] Autosomal recessive inheritance is the most common genetic form of ID, with high recurrence risk in populations with parental consanguinity.[8]

In 2016 we performed a field study in a highly inbred area of northeastern Brazil and identified a large consanguineous family with nine adult members affected with severe ID associated with disruptive behavior. We identified a homozygous deleterious variant in the inositol monophosphatase 1 gene (IMPA1), consisting of a 5-bp duplication leading to a frameshift and a premature stop codon, which cosegregated with the disorder in 26 genotyped family members.[9] More recently, a different loss-offunction mutation in the IMPA1 gene was found in six individuals with ID in a consanguineous family from Pakistan.[10]

Inositol monophosphatase (IMPAse) 1 dephosphorylates inositol monophosphate to generate inositol. IMPA1 enzyme is necessary for both de novo synthesis of inositol and inositol recycling from inositol polyphosphates.[11] Inositol is an essential metabolite because it is the precursor of membrane 
phospholipid phosphatidylinositol (PI) and its derivatives, inositol 1,4,5-trisphosphate and diacylglycerol, which are second messengers in important signaling pathways.[12] Therefore, dysregulation of the inositol cycle is implicated in many other human diseases, including cancer, diabetes and neurological diseases.[13] Among the two genes encoding for IMPase, IMPA1 is significantly more abundant in the brain than IMPA2.[14] Interest in IMPA1 function increased over the years, as it was recognized that this enzyme is inhibited by lithium, which is a mood stabilizer used for treatment of bipolar disorder.[13, 15, 16] Studies using animal models have attempted to understand the physiological consequences of IMPA1 inhibition by lithium with inconsistent results. These inconsistencies reflect the complex homeostasis of inositol, which depends on import, recycling and de novo synthesis pathways, and on the different roles of the related metabolites, phosphoinositols and phosphoinositides.[17, 18] Different studies suggested the existence of several pools of inositol in the brain and that the inhibition of IMPA1 affects a specific group of neurons and/or affects a specific metabolic phosphatidylinositol pathway.[17, 19, 20] In a recent study, it was shown that although lithium has little effect on steady-state levels of either inositol or membrane phosphoinositides, it markedly inhibits the rate of membrane phosphoinositides synthesis in mature cultured cortical neurons from rats.[18]

Two studies characterized the phenotypic effects in mice of mutations in IMPA1 gene in mice.[19, 21] A study by Cryns et al.[19] of a knockout IMPA1 mouse model revealed high embryonic lethality between post-coitum days 9.5 and 10.5 . Genotype analysis of 4-week-old offspring derived from heterozygous breeding revealed that only $2 \%$ instead of the expected $25 \%$ of Impa1 $1^{-/-}$mutants could be recovered at that time point. Interestingly, embryonic lethality could be reversed by inositol supplementation in the mothers' drinking water during the entire gestation period. Behavioral analysis of the $\operatorname{Impa1}^{-/-}$mice showed hyperactivity regardless of inositol supplementation. Adult $\operatorname{Impa1}^{-/-}$mice supplemented with inositol until weaning showed decreases in IMPase activity levels of $65 \%$ in the hippocampus and $40 \%$ in the frontal cortex and cerebellum compared to wild-type mice. However, inositol levels in the same brain regions were normal, which is reminiscent of other studies showing that IMPase inhibition by 
lithium does not affect inositol steady state.[18] Ohnishi et al.[21] screened an ethyl-nitrosourea mutant library for Impa1 ${ }^{-/-}$mutations and found a Thr95Lys missense mutation, which caused loss of IMPase activity. In agreement with previous results, homozygous progeny died perinatally but survival was also rescued by dietary inositol supplementation during gestation and the adults also showed hyperactivity. However, E18.5 embryos exhibited striking developmental defects, including hypoplasia of the mandible and asymmetric fusion of ribs to the sternum.

Understanding the interactions between networks of genes and proteins in relevant human models (e.g. patient-derived neural cells) is of paramount importance to clarify the molecular pathogenesis of ID and potentially develop therapeutic drugs.[22] Therefore, we used induced pluripotent stem cell (iPSC) technology to model IMPA1 deficiency in ID. In mouse brain, IMPA1 mRNA is localized at high levels in hippocampus (widely expressed in dentate gyrus) and frontal cortex.[14, 19, 21] We investigated the cellular defects of IMPA1 deficiency using hippocampal dentate gyrus-like neurons, pan-neuronal cells and astrocytes derived from iPSCs generated from ID patients and neurotypical controls. Here we show for the first time that IMPA1 mutation affects the ability of neural progenitor stem cells to differentiate into neurons but not into astrocytes.

\section{MATERIALS AND METHODS}

\section{Family ascertainment}

Affected and healthy family members from a small isolated community in an impoverished area of Northeastern Brazil were clinically evaluated in their hometown as part of a larger project on prospection of neurologic disorders in highly inbred areas of the country. After obtaining written consent from the parents or legal guardians, blood and/or skin biopsy samples were collected and a pedigree was constructed on the basis of family information and genotyping (Figure 1A). A blood sample was collected 
from an individual from another city in the study region as an external control. The data sampling protocol and the informed consent procedure were reviewed and approved by the National Committee for Ethics in Research (CONEP; Process Number, 55833216.2.1001.5187; Brazil). The studies performed at the Salk Institute were approved by the Institutional Review Board (IRB protocol number: 09.0003).

\section{iPSC reprogramming and pluripotency verification}

The iPSC lines were reprogrammed in the Human Genome and Stem Cells Research Center (University of São Paulo) from peripheral blood CD71-positive cells (patients and external control) and primary fibroblasts from skin (familial control). CD71-positive cells were sorted using magnetic labeled antibody (Miltenyi) following the manufacturer's instructions. The reprogramming protocol was performed with an episomal vectors system (pCXLE-hOCT3/4-shP53-F, Addgene plasmid 27077; pCXLE-hSK, Addgene plasmid 27078; pCXLE-hUL, Addgene plasmid 27080) according to Okita et al.[23] and using the Amaxa human CD34+ cells Nucleofection Kit (Lonza), following the manufacturer's recommendations.

Three days after nucleoporation, cells were seeded on irradiated murine embryonic fibroblasts (Millipore, A24903) in embryonic stem cell medium (Dulbecco's modified Eagle's medium (DMEM)/F12 supplemented with $2 \mathrm{mM}$ GlutaMAX-I, $0.1 \mathrm{mM}$ non-essential amino acids, $100 \mu \mathrm{M}$ 2-mercaptoethanol, $20 \%$ of knockout serum replacement (all provided by Life Technologies), $10 \mathrm{ng} / \mathrm{mL}$ of bFGF (Peprotech), $0.25 \mathrm{mM} \mathrm{NaB}, 0.5 \mathrm{mM}$ valproic acid, $2 \mu \mathrm{M}$ thiazovivin, $0.5 \mu \mathrm{M}$ PD 0325901 and $2 \mu \mathrm{M}$ SB 431542; all provided by Tocris Bioscience). Typical iPSC colonies were transferred to human embryonic stem cell (hESC)-qualified Matrigel (Corning)-coated $60 \mathrm{~mm}$ petri dishes (Corning) and cultured in Essential 8 Medium (Gibco) with $100 \mu \mathrm{g} / \mathrm{mL}$ normocin (InvivoGen). After reprogramming, iPSC colonies were selected for further quality control and validation by verifying the absence of integration of episomal reprogramming plasmids, normal karyotype, and expression of pluripotency markers (OCT4 and SSEA4). PCR was performed to detect the presence of Episomal iPSC Reprogramming Vectors in reprogrammed iPSC colonies by endpoint PCR according to the Epi5 Episomal iPSC Reprogramming Kit User Guide (Thermo Scientific). Using total DNA extracted from cultured cells, MLPA analysis was performed with 
subtelomeric kits (P036 and P070; MRC-Holland) to detect chromosomal imbalances as previously described.[24] Standard G-banding karyotype analyses were performed by Cell Line Genetics (Madison, WI). Cell lines were checked periodically for mycoplasma contamination by ELISA.

\section{Sanger sequencing}

The disorder-causing mutation (c.489_493dupGGGCT) within IMPA1 was sequenced by Sanger. A 193bp fragment harboring the candidate mutation was amplified using 5'-CCATGAACAGGAATGCAAAA$3^{\prime}$ as forward primer and 5'-GGGATACAAATGCCCTCTTC-3' as reverse primer. The reaction products were analyzed with an ABI 3730 DNA Analyzer (Applied Biosystems, Carlsbad, CA, USA), and the results analyzed using Sequencher 5.0 (Gene Codes, Ann Arbor, MI).

\section{Neurons and astrocytes differentiation and co-culture}

Hippocampal dentate gyrus-like neuronal precursors cell (DG-NPC) generation and dentate gyrus-like neuronal differentiation were performed as previously described.[25] Pan NPC generation and neuronal differentiation were performed as previously described.[26] Glial precursors cell (GPCs) generation and astrocyte differentiation were performed as previously described.[27] Human fetal astrocytes isolated from cerebral cortex (ScienCell \#1800, Carlsbad, USA) were cultured in DMEM-F12/Glutamax (Gibco) with $10 \%$ FBS; after two days, DG-NPCs were seeded on astrocytes and differentiated using DMEM/F12 supplemented with N2, B27, $20 \mathrm{ng} / \mathrm{ml}$ BDNF (Peprotech), $1 \mathrm{mM}$ dibutyryl-cAMP (Sigma), $200 \mathrm{nM}$ ascorbic acid (Sigma), $1 \mu \mathrm{g} / \mathrm{ml}$ laminin and $620 \mathrm{ng} / \mathrm{ml}$ Wnt3a (R\&D) and 2\% FBS. DMEM/F12 Glutamax media contains $70 \mu \mathrm{M}$ myo-inositol. iPSCs and NPCs at passage 3 were treated with $1 \mathrm{mM}$ myo-inositol throughout the neuronal differentiation to investigate the effect of inositol supplementation on phenotype rescue in vitro. 


\section{Lentiviral vector transduction}

To generate a stable shIMPA1 control line, NPCs from controls at P6 were transduced with lentivirus expressing shRNAs and selected for puromycin resistance for 3 successive media changes using 0.5 $\mu \mathrm{g} / \mathrm{ml}$ concentration. The shIMPA1 (sc-61115-V) or shControl containing an universal scrambled sequence (sc-108080) virus were purchased from Santa Cruz.

\section{Immunocytochemistry}

Cells were fixed in $4 \%$ paraformaldehyde for $20 \mathrm{~min}$ at room temperature, permeabilized with $0.5 \%$ Triton-X100 in PBS for $15 \mathrm{~min}$ at room temperature, and blocked in PBS containing $0.5 \%$ Triton-X100 and $10 \%$ horse serum for $1 \mathrm{~h}$ before incubation with primary antibody overnight at $4{ }^{\circ} \mathrm{C}$ (Supplementary Table 1). After 3 washes with PBS, cells were incubated with secondary antibodies for 1 hour at room temperature. Nuclei were stained with DAPI incubation for $10 \mathrm{~min}$. Fluorescent signals were detected using a Zeiss inverted microscope and images were processed and analyzed with Zen and ImageJ/Fiji.

\section{Western blotting}

For Western blotting, approximately $3 \times 10^{6}$ NPCs were lysed on ice for 5 min with RIPA buffer (Thermo Scientific) containing protease and phosphatase inhibitors (Thermo Scientific). Lysates were clarified by centrifugation $\left(15,000 \mathrm{rcf}\right.$ for $15 \mathrm{~min}$ at $\left.4^{\circ} \mathrm{C}\right)$, and Western immunoblotting was performed following standard procedures using the iBlot gel and transfer system (Life Technologies). Briefly, blots were incubated in primary antibodies anti-IMPA1 (1:1000, Abcam, ab202131) and anti-GAPDH (1:5000, Acris, ACR001P) and anti- $\beta$-Actin (1:30000, Abcam, ab49900) overnight at $4{ }^{\circ} \mathrm{C}$ and, following washes, they were incubated in horseradish peroxidase coupled secondary antibodies for $1 \mathrm{~h}$ at room temperature. All membranes were visualized using ECL (Thermo Scientific, 34080) and exposure to autoradiography film (Denville Scientific Inc. E3018). 


\section{RNA extraction, sequencing and analysis}

For whole transcriptome analysis, DG-NPCs from two clones of each sample (three patients and two controls) were treated with $1 \mathrm{mM}$ myo-inositol for 72 hours. Inositol-treated and untreated NPCs were harvested for RNA isolation. Two-week-old neurons infected with Prox $1::$ eGFP lentivirus at eight days differentiation were FACS sorted for $\operatorname{Prox}^{+} / \mathrm{PSA}_{\mathrm{NCAM}}{ }^{+}$cells for further RNA extraction. Total RNA was extracted from approximately $3 \times 10^{6}$ cells using RNA-Bee (Qiagen) according to the manufacturer's instructions. RNA-Seq libraries were prepared using the TruSeq Stranded mRNA Sample Prep Kit following the manufacturer's instructions (Illumina) and reverse transcribed into cDNA with SuperScript II reverse transcriptase (Invitrogen). Stranded cDNA libraries were generated according to Illumina's procedures. Total (mRNA) RNA-Seq libraries were sequenced as single-end 50 base pairs using the Illumina ${ }^{\circledR}$ HiSeq 2500 platform following the manufacturer's specifications. Low-quality ends and adapter removal/trimming were performed using cutadapt. Trimmed reads were mapped using STAR and assigned to genes with feature counts. Normalized counts and differential expression and statistics were calculated using the DESeq2 R package. Normalized count numbers were processed using R software to plots for further analysis and visualization. Metascape software (http://metascape.org) was used to perform Gene Ontology (GO) enrichment analysis.

\section{Electrophysiology}

Whole-cell patch-clamp recordings were performed as previously described.[28] Briefly, neurons were recorded in an extracellular recording medium containing (in mM): $10 \mathrm{HEPES}, 4 \mathrm{KCl}, 2 \mathrm{CaCl}_{2}, 1 \mathrm{MgCl}_{2}$, $139 \mathrm{NaCl}, 10 \mathrm{D}$-glucose (osmolarity and $\mathrm{PH}$ adjusted to $310 \mathrm{mOsm}, \mathrm{pH}$ 7.4). Whole-cell patch-clamp recordings were performed from Prox $1::$ eGFP lentivirus transfected neurons, usually around five weeks starting differentiation. Patch electrodes were filled with internal solutions containing (in $\mathrm{mM}$ ): $130 \mathrm{~K}$ gluconate, $6 \mathrm{KCl}, 4 \mathrm{NaCl}, 10 \mathrm{Na}$-HEPES, 0.2 K-EGTA, 0.3 GTP, 2 Mg-ATP, 0.2 cAMP, 10 D-glucose, $0.15 \%$ biocytin and $0.06 \%$ rhodamine. Recordings were conducted at room temperature. 


\section{Cell cycle and apoptosis assays}

For cell cycle assay, cells were incubated with $10 \mu \mathrm{M}$ EdU for 2 hours, harvested and EdU-detected using Click-iT® Plus EdU Alexa Fluor® 488 Flow Cytometry Assay Kit (C10632, Thermo Fisher Scientific) following the manufacturer's instructions. For total DNA staining cells were stained with $100 \mu$ PBS +1 $\mu \mathrm{g} / \mathrm{ml}$ DAPI for 4 hours at $4^{\circ} \mathrm{C}$. Apoptotic cells were labeled using a FITC Annexin V kit (640905, BioLegend) according to the manufacturer's instructions. Cells were analyzed by flow cytometry (LSRII, BD Biosciences) and quantified using FlowJo software.

\section{Data analysis and statistics}

Experiments were repeated as independent duplicates to obtain averages per individual per group for further statistical analysis. All data values were presented as mean \pm SEM. For parametric datasets with two groups, unpaired Student's t-test was used to compare averages between two groups. Statistical tests and $\mathrm{p}$ values for individual experiments are stated in the figure legends, and $\mathrm{p}<0.05$ was considered statistically significant.

\section{RESULTS}

\section{IMPA1 mutation considerably modifies transcriptome profile of neuronal progenitor cells}

We previously identified the IMPA1 mutation in ID patients in a large consanguineous family from Northeastern Brazil.[9] The total frequency of consanguineous marriages in Brazil is about 15 times higher in the Northeast $(9.00 \%)$ than in the South $(0.62 \%),[29-31]$ and unfortunately this tradition continues.[32] To provide genetic counseling to this family, we genotyped 33 additional individuals, which identified 9 novel heterozygous individuals (Figure 1A). The data from this family that includes 28 consanguineous marriages, 59 genotyped individuals and nine ID patients show co-segregation of the variant with the disorder up to seven generations. However, the impact of IMPA1 loss-of-function in the human brain cells is not known. We took advantage of iPSC technology to model this particular familial 
ID in stem cells, neurons and astrocytes in a dish. The demographic and clinical information from the individuals from whom we collected samples for reprogramming is detailed in Figure 1B. The episomal reprogramming method [23] was used to generate iPSCs from three patients with mutated IMPA1 (Patients 1-3) and two healthy controls (Controls 1 and 2) (Figure 1C). Two clones from each iPSC line were confirmed positive for OCT4 and SSEA4 pluripotency markers (Figure 1D) and showed normal karyotype by standard G-banding and MLPA analysis (Figure 1E). The retention of IMPA1 mutation in iPSC lines was confirmed by Sanger sequencing (Supplementary Figure 1).

Considering that IMPA1 is highly expressed in the hippocampus and that the reduction in IMPAse activity in the mouse knockout model was highest in this brain region,[14, 19, 21] we chose to investigate the phenotypes of IMPA1 mutation in dentate gyrus-like neurons derived from ID patients' iPSCs. To do so, we differentiated NPCs from iPSCs following described methods[25] (Figure 2A). Briefly, patterned embryoid bodies were plated for one week and the dissociated rosettes formed homogeneous adherent monolayers of NPCs positive for the markers SOX2 and Nestin, with no significant difference from those derived from patients and controls (Figure 2B and 2C). The IMPA1 enzyme was undetectable in NPCs derived from patients with mutated IMPA1 by Western blot analysis (Figure 2D), confirming that the 5-bp duplication, which introduces a frameshift and a premature stop codon in IMPA1, abolishes protein expression. Therefore, cells derived from ID patients from this family constitute a good human model to study the molecular, cellular and metabolic consequences of IMPA1 deficiency.

We performed whole transcriptome analysis using mRNA sequencing to identify core molecular pathways underlying IMPA1 loss-of-function using two NPC clones from each individual at passages 2 or 3. Differential expression analysis was performed using the statistical R packages EdgeR and DESeq and resulted in a substantial list of 633 downregulated genes and 1,057 upregulated genes in NPCs derived from ID patients compared to controls (Bonferroni-corrected p-value $<0.05$ and $\log 2$ fold $>2$ ). The complete list appears in Supplementary Table 2. To further characterize the biological processes that may be affected in patients with mutated IMPA1, we investigated the functional annotations associated 
with the 1,690 differentially expressed genes (Figure 2E and 2F). GO analysis revealed significant enrichment for genes involved in multiple developmental processes including brain development and regionalization, cell fate commitment, extracellular structure organization and regulation of signaling receptor activity. Interestingly, GO analysis also suggests upregulation of the gliogenic pathway and downregulation of neuronal differentiation (Figure $2 \mathrm{E}$ and $2 \mathrm{~F}$ ). We found 52 of the differentially expressed genes involved in gliogenesis (GO:0042063; 39 upregulated genes), 77 genes involved in cell fate commitment (GO:0045165; 53 upregulated genes and 14 downregulated genes) and 149 genes involved in regulation of nervous system (GO:0051960; 96 upregulated genes and 53 downregulated genes) in NPCs from patients compared to controls (Supplementary Table 2). Detailed analysis of differentially expressed genes in the phosphatidylinositol signaling pathway showed increase in expression of inositol 1,4,5-trisphosphate receptors type 3 (ITPR3; fold change $=2.92 ; \mathrm{FDR}=4.23 \mathrm{e}-38$ ) and type $2(I T P R 2 ;$ fold change $=2.00 ;$ FDR $=0.002) \quad($ Supplementary Table 2$)$, which trigger calcium release from the endoplasmic reticulum.

Considering the large number of differentially expressed genes in IMPA1-mutated NPCs and that inositol supplementation rescued embryonic or perinatal lethality in the mouse knockout models, $[19,21]$ we tested whether $1 \mathrm{mM}$ inositol treatment for 72 hours could rescue gene expression. Surprisingly, inositol supplementation had no effect on gene expression in IMPA1-deficient NPCs (data not shown).

\section{IMPA1 loss-of-function specifically affects neuronal progenitor survival and proliferation}

A substantial number of upregulated genes in NPCs from ID patients compared to controls are involved in apoptosis and cell cycle control (Figure 2H and 2I), which are intimately linked to cellular processes [33]. For example, expression of CASP4 and CASP1 genes was increased by 5.6- and 4.2-fold $(\mathrm{P}<0.05)$ in IMPA1-mutated NPCs. These are inflammatory caspases involved in endoplasmic reticulum stressinduced apoptosis.[34, 35] We further identified important genes involved in cell cycle progression with altered expression in IMPA1-mutated NPCs (Figure 2I); most of these genes are associated with negative 
regulation of cyclin-dependent protein serine/threonine kinase activity involved in the G1/S transition.[36]

We observed changes in morphology (Figure 3A) and a low proliferation rate in NPCs derived from patients with mutated IMPA1 compared to controls in passages higher than P3. This observation was in agreement with the gene expression data suggesting increased apoptosis and modifications in cell cycle progression, as well as increased gliogenic potential. Therefore, we tested for defects in cell cycle using flow cytometry assay. Analysis of cell cycle progression of NPCs at P2 revealed a significant decrease in the proportion of cells in S phase in IMPA1-mutated NPCs compared to controls, even at this low passage (Figure 3B and 3C; Supplementary Figure 2). Apoptosis was measured in living cells using annexin V to detect phosphatidylserine localized on the outer leaflet of the plasma membrane by flow cytometry. A significant increase in the proportion of cells undergoing apoptosis was observed in NPCs derived from patients with mutated IMPA1 compared to controls at P2 and it was more significant after initiation of neuronal differentiation (Figure 3D and 3E; Supplementary Figure 2). We then asked if these defects were already present in iPSCs. Interestingly, no changes in cell cycle progression or increase in apoptosis were observed in IMPA1-mutated iPSCs compared to controls (Figures 3C and 3E; Supplementary Figure 2). Since the morphology of IMPA1-mutated NPCs changed to become flatter and more glial-like as the passage number increased, we differentiated the iPSC cohort into glial progenitors (GPCs). The results were similar to those obtained for iPSCs; no changes in cell cycle progression or apoptosis were observed in IMPA1 GPCs compared to controls (Figures 3C and 3E; Supplementary Figure 2). Altogether these results suggest that IMPA1 loss-of-function specifically affects neural progenitor cells that show defects in proliferation and survival.

\section{Neuronal differentiation is impaired by IMPA1 deficiency}

Neuronal differentiation from IMPA1-mutated NPCs was severely impaired (Figures 4A and 4B). After five weeks of differentiation, less than $20 \%$ of cells expressed the neuronal marker MAP2 (Figure 4B). To exclude the hypothesis that it is the defects in NPCs proliferation and increased apoptosis that impairs 
neuronal differentiation, the initial NPC number at passages 1 or 2 was increased by a factor of 2 or 4 . The results showed no improvement in the proportion of differentiated neurons, suggesting that IMPA1 deficiency also affects the neurogenic potential of NPCs. Immunofluorescence data obtained with confocal microscopy showed that the IMPA1-mutated dentate gyrus-like neurons that differentiated for five weeks had altered morphology compared to those derived from control NPCs. In particular, the IMPA1-mutated neurons exhibited less complexity of processes compared to controls (Figure 4A). Interestingly, although IMPA1 deficiency causes a drastic reduction in neuronal differentiation from progenitors, the few neurons that mature are active (Figures 4C, 4D and 4E). To confirm if this phenotype was specific to dentate gyrus differentiation or more general, iPSCs from controls and ID patients were differentiated into NPCs and neurons according to the pan-neuronal differentiation method described previously.[26] As for DG neurons, the pan neurons derived from patients with mutated IMPA1 showed a low proportion of developing neurons compared with neurons derived from control individuals (Supplementary Figure 3).

Transcriptome analysis using mRNA sequencing was performed in two clones from each patient- and control-derived two-week-old neurons. The cultures were FACS sorted for Prox1-positive and PSANCAM-positive markers of dentate gyrus neurons to ensure that only neurons were analyzed. We found 1,346 differentially expressed genes (661 upregulated and 685 downregulated; Bonferroni-corrected pvalue $<0.05$ and $\log 2$ fold $>2$ ) in IMPA1-mutated neurons compared to controls (Supplementary Table 3). GO analysis revealed significant enrichment for genes involved in different developmental programs, extracellular matrix organization and neuropeptide signaling pathways (Figure 4F). We found 43 genes involved in cell surface receptor signaling that were differentially expressed in neurons derived from patients compared to controls (GO:1905114; 43 upregulated genes; Supplementary Table 3), including genes involved in the canonical Wnt signaling pathway (e.g., DKK1/2, WNT1/6/10A, LGR5/6 and BMP4). Interestingly, a significant proportion of genes implicated in gliogenesis were upregulated in IMPA1-deficient neurons (GO:0042063; 29 upregulated genes), which include important glial proteins 
such as GFAP (fold change $=3.94 ; \mathrm{FDR}=0.0004)$ and $S 100 B($ fold change $=2.15 ; \mathrm{FDR}=0.004)$. Therefore, we concluded that genes related to gliogenesis were upregulated in patients DG neurons as compared to controls.

Inositol supplementation during gestation rescued embryonic or perinatal lethality in two mouse models of IMPA1 deficiency.[19, 21] We tested if inositol addition to the media throughout neuronal differentiation from NPCs at P3 would increase the proportion of differentiating neurons and observed no difference 10 days post-differentiation (Figure 4G). However, when inositol supplementation was done throughout differentiation of iPSCs into NPCs and neurons, we observed a significant improvement in neurogenesis (Figure 4G). This result suggests that although IMPA1 deficiency is not detrimental for iPSC, it is critical for generation of NPCs and neurons. It was also an indication that the neurogenic deficit of ID patient-derived NPCs was due to IMPA1 mutation and not to a combination of risk gene mutations. To test if the opposite was true, NPCs from the unrelated control cell line were transduced with lentivirus expressing shIMPA1 or a scrambled control and selected for stable populations using the puromycin antibiotic. IMPA1 protein level detected by Western blotting was reduced by approximately $75 \%$ in NPCs expressing shIMPA1 compared to controls (Figure $4 \mathrm{H}$ ). We observed a significant decrease in the proportion of neurons in the shIMPA1 condition (Figure 4I). Altogether, these results show that IMPA1 deficiency decreases the capacity of iPSC and NPCs to differentiate into neurons.

\section{IMPA1 deficiency does not affect astrocyte differentiation from GPCs}

Considering the high number of genes upregulated in NPCs and neurons that encode components of the gliogenic pathway, and that GPCs do not show any defect in cell cycle or increased apoptosis, we analyzed GPC and astrocyte differentiation in detail. No difference was observed between cells derived from patients with mutated IMPA1 and controls concerning the expression of specific markers for GPC and astrocyte differentiation (Figure 5A-5D), suggesting that the defects observed were specific to NPCs and neuronal differentiation. However, to exclude that astrocytes that differentiated at the same time in 
neuronal cultures could be responsible for arrested neuronal differentiation, we performed co-culture of primary astrocytes with differentiating NPCs into dentate gyrus-like neurons. After 10 days, the cocultures were analyzed and there was no improvement in survival or maturation of neurons deficient for IMPA1 (Figure 5E). These results suggest that the neuronal defects are cell-autonomous.

\section{DISCUSSION}

In this study, we successfully combined field investigation in the rural and isolated environment where the patients live with state-of-the-art iPSC technology to investigate the molecular and cellular pathways implicated in IMPA1 loss-of-function and severe ID. We show that, although IMPA1 deficiency did not affect iPSCs, GPCs and astrocyte differentiation, it had a dramatic effect in NPCs, causing cell cycle arrest, apoptosis and poor neurogenic capability. Co-culture with primary astrocytes did not improve neurogenesis suggesting that the neuronal defects are cell-autonomous and consequence of mutation. However, the few progenitor cells that differentiated into neurons showed normal activity. Interestingly, we observed a significant improvement in neurogenesis with inositol supplementation during all steps of neuronal differentiation starting from iPSCs, but not when started from NPCs. Therefore, the critical period for in vitro differentiation of hippocampal DG-like neurons from IMPA1-mutated patients is iPSCs to NPCs. Our results are reminiscent of those obtained in $\operatorname{Impa1}^{-/-}$mice models showing that inositol supplementation during gestation period rescued embryonic or perinatal lethality. These results highlight the importance of inositol in the early embryonic development and suggest that inositol deficiency, either by deficient synthesis, recycling or transport is detrimental for normal development.

Transcriptome analysis showed that NPCs and neurons derived from ID patients had extensive dysregulation of gene expression affecting pathways necessary for neurogenesis, such as Wnt and GPCR signaling pathways.[37, 38] In addition, NPCs derived from patients with mutated IMPA1 revealed increased expression of 39 genes involved in gliogenesis and significant overexpression of Hes 3 or Hes5, which were shown to inhibit neuronal differentiation and maintain neural stem cells in the embryonic 
brain.[39-41] As passages increased, IMPA1-deficient NPCs stopped proliferating and adopted a more gliogenic progenitor phenotype. Interestingly, transcriptome analysis of two-week-old neurons derived from IMPA1-mutated patients revealed increased expression of important glial markers (S100B, S100A2, PAX6, GFAP, ALDH1L1 and CD40).

A previous study reported upregulation of oxidative phosphorylation and other mitochondria-related genes in the frontal cortex of knockout IMPA1 and SMIT1 mice suggesting that deficient inositol recycling, de novo synthesis and transport affect mitochondrial functions.[42] Surprisingly, transcriptome analysis of NPCs and neurons derived from IMPA1-mutated patients didn't show significant difference in mitochondrial genes compared to controls. However, it is possible that mitochondria is implicated in the apoptotic phenotype of NPCs derived from patients, since they show increased expression of ITPR3 and ITPR2. These genes encode endoplasmic reticulum receptors that release calcium to the cytoplasm upon stimulation by IP3 (inositol 1,4,5-trisphosphate). In physiological concentrations, calcium release from ITPR3 stimulates mitochondrial respiration. However, when calcium release is prolonged and excessive it causes mitochondrial calcium overload that leads to a subsequent decrease in mitochondrial membrane potential, reactive oxygen species accumulation, senescence and apoptosis.[43-45] Therefore, a mitochondrial dysfunction cannot be ruled out as an important pathophysiological mechanism underlying the association between IMPA1 deficiency and NPCs apoptosis and deficient neurogenic potential.

The majority of studies involving inositol pathway have been focused on what are the in vivo downstream consequences of IMPA1 knockout and chronic Li treatment, an uncompetitive inhibitor of IMPA1, evaluating inositol depletion reduced re-synthesis of phosphoinositides, accumulation of phosphoinositols and/or attenuated inositol turnover. In the present study, we used genetic tools combined with human cellular models and transcriptome profiling to understand the role of the IMPA1 loss-of-function in ID and showed a specific importance of the inositol cycle in human neuronal development. This association was validated by rescuing the phenotype with myo-inositol supplemented media during differentiation of 
patient derived-iPSCs into neurons and by the reduction of neurogenic potential in control NPCs expressing shIMPA1. 


\section{CONFLICT OF INTEREST}

The authors declare no conflict of interest.

\section{ACKNOWLEDGMENTS}

This manuscript is dedicated to the family involved in this study. This study was financially supported by the CEPID-FAPESP (2013/08028-1), INCT/FAPESP (2014/50931-3) and FAPESP (Process Number: 2019/18469-1, 2017/19877-0 and 2016/09618-5). This work was supported in part by the National Cancer Institute (Grant No. P30 CA014195) and the National Institutes of Health (Grant No. R01AG05651) and by the National Cooperative Reprogrammed Cell Research Groups (NCRCRG) (Grant No. U19 MH106434). The Gage laboratory is supported in part by the Leona M. and Harry B. Helmsley Charitable Trust Grant No. 2017-PG-MED001, the JPB Foundation, Annette C. Merle-Smith, and the Robert and Mary Jane Engman Foundation. We also thank the Salk core facilities. This work was supported in part by the Next Generation Sequencing Core (NGS) Facility of the Salk Institute with funding from NIH-NCI CCSG: P30 014195, the Chapman Foundation and the Helmsley Charitable Trust. 


\section{REFERENCES}

1. American Psychiatric Association. Diagnostic and Statistical Manual of Mental Disorders. American Psychiatric Association: Washington, DC, USA, 2000.

2. Durkin M. The epidemiology of developmental disabilities in low income countries. Ment Retard Dev Disabil Res Rev. 2002;8:206-211.

3. Emerson E. Poverty and people with intellectual disabilities. Ment Retard Dev Disabil Res Rev. 2007; 13:107-113.

4. Durkin MS, Hasan ZM, Hasan KZ. Prevalence and correlates of mental retardation among children in Karachi, Pakistan. Am J Epidemiol. 1998;147:281-288.

5. Leonard H, Wen X. The epidemiology of mental retardation: challenges and opportunities in the new millennium. Ment Retard Dev Disabil Res Rev. 2002;8:117-134.

6. Vissers LE, Gilissen C, Veltman JA. Genetic studies in intellectual disability and related disorders. Nat Rev Genet. 2016;17:9-18.

7. Ropers HH. Genetics of intellectual disability. Curr Opin Genet Dev. 2008;18:241-250.

8. Musante L, Ropers HH. Genetics of recessive cognitive disorders. Trends Genet. 2014;30:32-39.

9. Figueiredo T, et al. A homozygous loss-of-function mutation in inositol monophosphatase 1 (IMPA1) causes severe intellectual disability. Mol Psychiatry. 2016;21:1125-1129.

10. Harripaul R, et al. Mapping autosomal recessive intellectual disability: combined microarray and exome sequencing identifies 26 novel candidate genes in 192 consanguineous families. Mol Psychiatry. 2017;23:973-984. 
11. Resnick AC, Saiardi A. Inositol polyphosphate multikinase: metabolic architect of nuclear inositides. Front Biosci. 2008;13:856-866.

12. Berridge MJ, Bootman MD, Roderick HL. Calcium signalling: dynamics, homeostasis and remodelling. Nat Rev Mol Cell Biol. 2003;4:517-529.

13. Hakim S, et al. Inositol polyphosphate phosphatases in human disease. Curr Top Microbiol Immunol. 2012;362:247-314.

14. Ohnishi T, et al. Spatial expression patterns and biochemical properties distinguish a second myoinositol monophosphatase IMPA2 from IMPA1. J Biol Chem. 2007;282:637-646.

15. Berridge MJ, Downes CP, Hanley MR. Neural and developmental actions of lithium: a unifying hypothesis. Cell. 1989;59:411-419.

16. Atack JR, Broughton HB, Pollack SJ. Inositol monophosphatase--a putative target for Li+ in the treatment of bipolar disorder. Trends Neurosci. 1995;18:343-349.

17. Sade Y, et al. IP3 accumulation and/or inositol depletion: two downstream lithium's effects that may mediate its behavioral and cellular changes. Trans1 Psychiatry. 2016;6:e968.

18. Saiardi A, Mudge AW, Lithium and fluoxetine regulate the rate of phosphoinositides synthesis in neurons: a new view of their mechanisms of action in bipolar disorder. Transl Psychriatry. 2018;8:175.

19. Cryns $\mathrm{K}$, et al. IMPA1 is essential for embryonic development and lithium-like pilocarpine sensitivity. Neuropsychopharmacology. 2008;33:674-684. 
20. Fisher SK, Novak JE, Agranoff BW. Inositol and higher inositol phosphates in neural tissues: homeostasis, metabolism and functional significance. J Neurochem 2012;82:736-754.

21. Ohnishi $\mathrm{T}$, et al. Defective craniofacial development and brain function in a mouse model for depletion of intracellular inositol synthesis. J Biol Chem. 2014;289:10785-10796.

22. Ropers HH. Genetics of early onset cognitive impairment. Annu. Rev. Genomics Hum Genet. 2010;11:161-187.

23. Okita K, et al. An efficient nonviral method to generate integration-free human-induced pluripotent stem cells from cord blood and peripheral blood cells. Stem Cells. 2013;31:458-466.

24. Jehee FS, et al. Using a combination of MLPA kits to detect chromosomal imbalance in patients with multiple congenital anomalies and mental retardation is a valuable choice for developing countries. Eur J Med Gene. 2001;54:425-432.

25. Yu DX, et al. Modeling hippocampal neurogenesis using human pluripotent stem cells. Stem Cell Rep. 2014;2: 295-310.

26. Marchetto MC, et al. Altered proliferation and networks in neural cells derived from idiopathic autistic individuals. Mol Psychiatry. 2017;22:820-835.

27. Santos R, et al. Differentiation of inflammation-responsive astrocytes from glial progenitors generated from human induced pluripotent stem cells. Stem Cell Rep. 2017;8:1757-1769.

28. Stern S, et al. Neurons derived from patients with bipolar disorder divide into intrinsically different sub-populations of neurons, predicting the patients' responsiveness to lithium. Mol Psychiatry. 2018;23:1453-1465.

29. Freire-Maia N. Inbreeding in Brazil. Am J Hum Genet. 1957;9:284-298. 
30. Freire-Maia N. Genetic effects in Brazilian populations due to consanguineous marriages. Am J Med Genet. 1989;35:115-117.

31. Fonseca LG, Freire-Maia N. Further data on inbreeding levels in Brazilian populations. Social Biol. $1970 ; 17: 324-328$.

32. Weller M, et al. Consanguineous unions and the burden of disability: a population-based study in communities of Northeastern Brazil. Am J Hum Biol. 2012; 24:835-840.

33. Meikrantz W, Schlegel R. Apoptosis and the cell cycle. J Cell Biochem. 1995;58:160-174.

34. Hitomi $\mathrm{J}$, et al. Involvement of caspase-4 in endoplasmic reticulum stress-induced apoptosis and Abeta-induced cell death. J Cell Biol. 2004;165:347-356.

35. Sakamaki K, Satou Y. Caspases: evolutionary aspects of their functions in vertebrates. J Fish Biol. 2009:74:727-753.

36. Engeland K. Cell cycle arrest through indirect transcriptional repression by p53: I have a DREAM. Cell Death Differ. 2018;25:114-132.

37. Doze VA, Perez DM. G-protein-coupled receptors in adult neurogenesis. Pharmacol Rev. 2012;64:645-675.

38. Varella-Nallar L, Inestrosa NC. Wnt signaling in the regulation of adult hippocampal neurogenesis. Front Cell Neurosci. 2013;7:100.

39. Ishibashi M, et al. Persistent expression of helix-loop-helix factor HES-1 prevents mammalian neural differentiation in the central nervous system. EMBO J. 1994;13:1799-1805. 
40. Hirata $\mathrm{H}$, et al. Generation of structurally and functionally distinct factors from the basic helix-loophelix gene Hes3 by alternative first exons. J Biol Chem. 2000:275:19083-19089.

41. Ohtsuka T, et al. Roles of the basic helix-loop-helix genes Hes1 and Hes5 in expansion of neural stem cells of the developing brain. J Biol Chem. 2001; 276:30467-30474

42. Toker L, et al. Inositol related gene knockouts mimic lithium's effect on mitochondrial function. Neuropsychopharmacology. 2014;39:319-328.

43. Wiel C, et al. Endoplasmic reticulum calcium release through ITPR2 channels leads to mitochondrial calcium accumulation and senescence. Nat Commun. 2014;5:3792.

44. Llorente-Folch I, et al. The regulation of neuronal mitochondrial metabolism by calcium. J Physiol. 2015;593:3447-3462.

45. Marchi S, et al. Endoplasmic Reticulum-Mitochondria Communication Through $\mathrm{Ca}^{2+}$ Signaling: The Importance of Mitochondria-Associated Membranes (MAMs). Adv Exp Med Biol. 2017;997:49-67. 


\section{FIGURE LEGENDS}

Figure 1. Genealogy and clinical features of individuals from a Brazilian family with IMPA1 mutation causing intellectual disability. (A) Seven generation family pedigree with 28 consanguineous marriages showing 59 genotyped individuals. Individuals with ID and heterozygous are represented by filled and half-filled symbols, respectively. Genotyped individuals are number coded; underlined code represents the individuals genotyped before[9] and red code represents individuals from whom iPSCs were generated. (B) Clinical and demographic information. (C) Schematic representation of episomal reprogramming. Scale bar, $1000 \mu \mathrm{m}$. (D) Representative images of iPSC colonies showing expression of pluripotency markers (OCT4 and SSEA4). Scale bar, $100 \mu \mathrm{m}$. (E) Representative examples of chromosome G-banding and MLPA analysis of iPSC clones showing normal karyotype.

Figure 2. Dentate gyrus patterned NPCs from patients with mutated IMPA1 showed conspicuous alterations in transcriptome profile. (A) Schematic representation of NPCs generation from iPSCs. (B) Quantification of the proportion of Sox2-positive and Nestin-positive in NPCs derived from controls and patients. (C) Immunolabeling for the neuronal precursor markers Sox2 and Nestin. Results are presented as mean \pm SEM. (D) Western blot analysis confirming that IMPA1 mutation causes loss of expression of IMPA1 enzyme in NPCs from IMPA1 mutated-patients. SDS gel electrophoresis was performed using 20 $\mu \mathrm{g}$ protein per lane. (E, F) Gene ontology (GO) analysis for all genes differentially expressed in IMPA1mutated patients NPCs versus control NPCs (Bonferroni-corrected P-value < 0.05 and $\log 2$ fold $>2$ ). (G) Heat map of the normalized gene expression involved in inositol cycle. (H) Genes involved in apoptosis with altered expression in IMPA1-mutated NPCs versus controls (Bonferroni-corrected p-value $<0.05$ and $\log 2$ fold $>2$ ). (I) Genes involved in cell cycle progression with altered expression in IMPA1-mutated NPCs versus controls.

Figure 3. NPCs, but not iPSCs or GPCs, derived from patients with mutated IMPA1 show defects in proliferation and survival. (A) Bright field images of NPCs at P3 showing a glial-like morphology in IMPA1-mutated patients NPC lines. Scale bar, $50 \mu \mathrm{m}$. (B) Representative flow cytometry plots of NPCs at P2 showing percentages of cells at different cell cycle phases. (C) Quantification of the cell cycle analysis of NPCs, iPSCs and GPCs represented as mean \pm SEM (** $\mathrm{p}=0.0058)$. (D) Representative flow cytometry plots of NPCs at P2 showing in Q1 necrotic cells, in Q2 early apoptotic cells, in Q3 late 
apoptotic cells and in Q4 live cells. (E) Quantification of cell death of NPCs, neurons (3 days of differentiation), iPSCs and GPCs represented as mean $\pm \operatorname{SEM}(* * * \mathrm{p}<0.0001 ; * * \mathrm{p}=0.0015)$.

Figure 4. Neuronal differentiation is impaired by IMPA1 deficiency. (A) Representative images of Map2 immunostainings of 5-week neuronal differentiation. (B) Quantification of Map2 positive cells in IMPA1 patients-derived neurons compared to controls after 5 weeks of differentiation represented as mean \pm SEM (*** $\mathrm{p}<0.0001)$. (C-E) Patch clamp recordings showing that neurons derived from IMPA1mutated patients have evoked and spontaneous action potentials. (C) Representative traces are shown of spontaneous activity. Cells were held in current clamp mode with a current injection that is close to 0 to have the membrane potential near $-45 \mathrm{mV}$ and the spontaneous action potentials were recorded and are shown in the trace. (D) Representative traces are shown of the evoked potentials acquired in current clamp mode. Cells were typically held in current clamp mode near $-60 \mathrm{mV}$ with a steady holding current, and current injections were given starting $12 \mathrm{pA}$ below the steady holding current, in $3 \mathrm{pA}$ steps of $400 \mathrm{~ms}$. (E) Representative traces are shown for the sodium/potassium currents while holding the cells in voltage clamp mode at $-60 \mathrm{mV}$ and briefly depolarizing the membrane potential to different test potentials. The downward deflections are inward sodium currents, while the upward deflections are outward potassium currents. (F) GO analysis for all genes differentially expressed in neurons from patients with mutated IMPA1 versus control neurons (Bonferroni-corrected p-value $<0.05$ and $\log 2$ fold $>2$ ). (G) Phenotype rescue by myo-inositol treatment of patient cells. Representative fluorescence images of immunostainings and quantifications of neurons expressing Tuj1 derived from iPSCs and NPCs treated with $1 \mathrm{mM}$ myo-inositol (10 days post-differentiation). (H-I) IMPA1 knockdown using lentivirus expressing shRNA. (H) Western blot analysis of stable cell populations expressing shIMPA1 and nonsilencing shSCR (scrambled control sequence). SDS gel electrophoresis was performed using $10 \mu \mathrm{g}$ protein per lane. (I) Quantification and representative fluorescence images of immunostainings of neurons expressing Tuj1 derived from NPCs expressing shRNA and shSCR (10 days post-differentiation).

Figure 5. GPC and astrocyte differentiation is not affected by IMPA1 deficiency. (A-B) Representative fluorescence images of immunostainings for GPCs expressing Nestin, A2B5 and NFIA and quantification of the proportion of Nestin-, A2B5- and NFI-positive GPCs derived from controls and patients represented as mean \pm SEM. (C-D) Representative fluorescence images of immunostainings for astrocytes expressing S100 $\beta$, GFAP and CD44 and quantification of the proportion of S100 $\beta$, GFAP and 
CD44-positive in astrocytes derived from controls and patients represented as mean \pm SEM. (E) Representative images of MAP2-expressing neurons derived from controls and patients co-cultured with S100ß-expressing primary human astrocytes.

\section{SUPPLEMENTARY LEGENDS}

Supplementary Figure 1. Retention of IMPA1 mutation in iPSC lines. Sanger sequencing electropherograms showing the IMPA1 5-bp microduplication in iPSC clones derived from ID patients.

Supplementary Figure 2. (A-C) Cell line distribution of cell cycle data presented in Figure 3C represented as mean \pm SEM. (D-G) Cell line distribution of apoptosis data presented in Figure $3 \mathrm{E}$ represented as mean $\pm \mathrm{SEM}$.

Supplementary Figure 3. Pan-neurons derived from IMPA1-mutated and control iPSCs. (A) Bright field images of pan-neurons with two weeks post-differentiation. Scale bar, $50 \mu \mathrm{m}$. (B) Representative fluorescent images of immunostainings for pan-neurons with 3 weeks post-differentiation expressing Tuj1.

Supplementary Table 1. List of antibodies used in this study.

Supplementary Table 2. Differential gene expression list of IMPA1-mutated NPCs versus control NPCs and Gene Ontology (GO) enrichment analysis. Bonferroni-corrected p-value $<0.05$ and $\log 2$ fold $>2$.

Supplementary Table 3. Differential gene expression list of IMPA1-mutated neurons versus control neurons with two weeks post-differentiation and Gene Ontology (GO) enrichment analysis. Bonferronicorrected p-value $<0.05$ and $\log 2$ fold $>2$. 


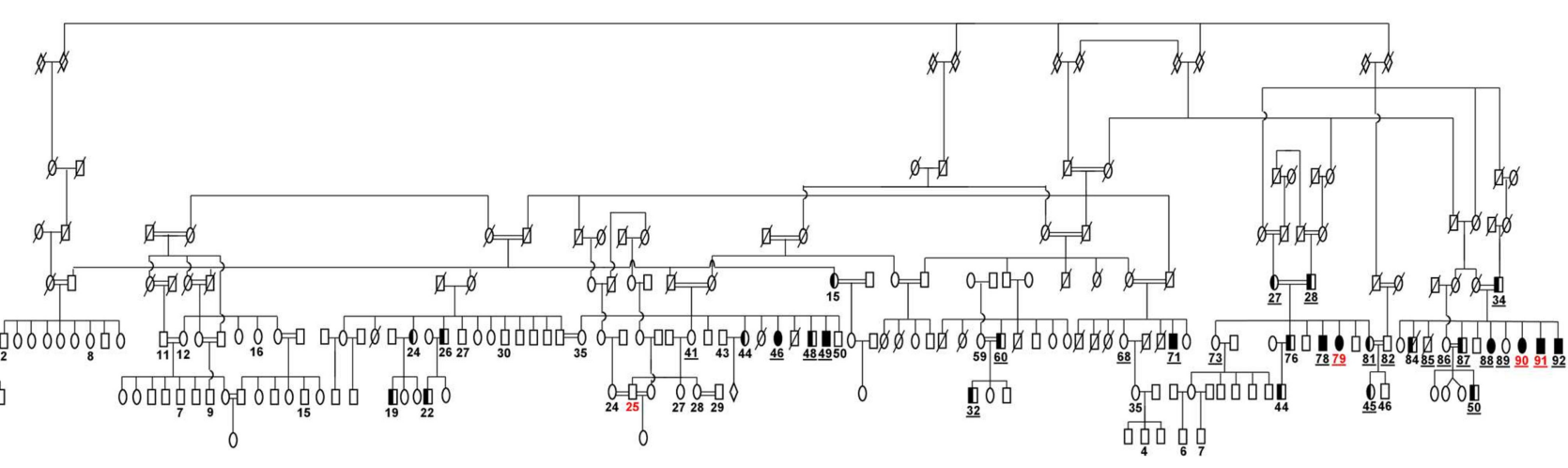

\begin{tabular}{|c|c|c|c|c|c|c|c|c|}
\hline Individuals & Genealogy & Age & Sex & ID & $\begin{array}{l}\text { Attention } \\
\text { Deficit }\end{array}$ & Behavior & Others & Medication \\
\hline Patient 1 & V-79 & 52 & $\mathrm{~F}$ & Mild & Yes & $\begin{array}{l}\text { Primary mood disorder } \\
\text { with prominent } \\
\text { hallucinations }\end{array}$ & & No \\
\hline Patient 2 & V-90 & 51 & M & Severe & Yes & Paranoid, Agitated & & No \\
\hline Patient 3 & V-91 & 60 & $\mathrm{~F}$ & Severe & Yes & Paranoid, Agitated & $\begin{array}{l}\text { Postural tremor, } \\
\text { bradykinesia }\end{array}$ & No \\
\hline Control 1 & VI-25 & 43 & M & No & No & Neurotypical & & No \\
\hline Control 2 & External & 38 & F & No & No & Neurotypical & & No \\
\hline
\end{tabular}

C

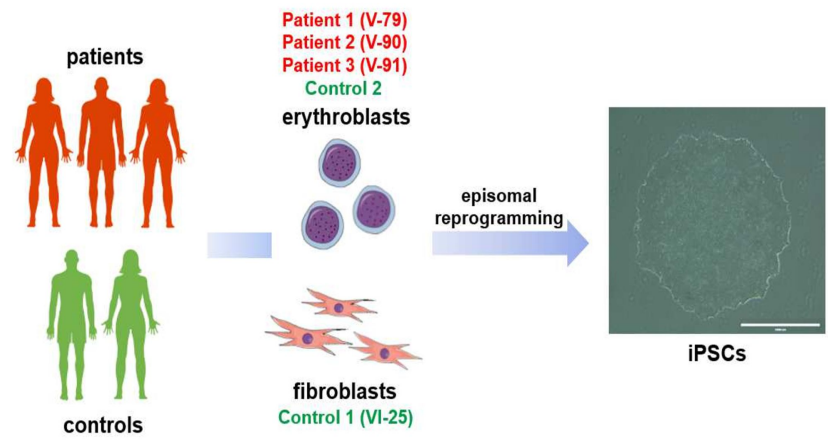

E

\section{Control 1}

If 10 is in

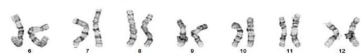

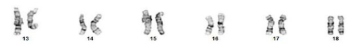

t.8 25 3.

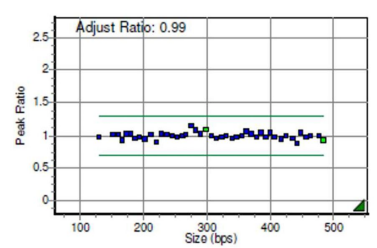

\section{Patient 3}

) 36703120

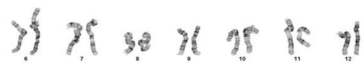

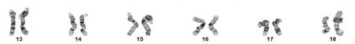

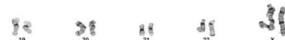

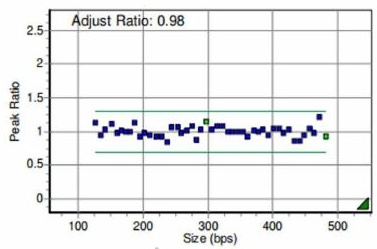

D

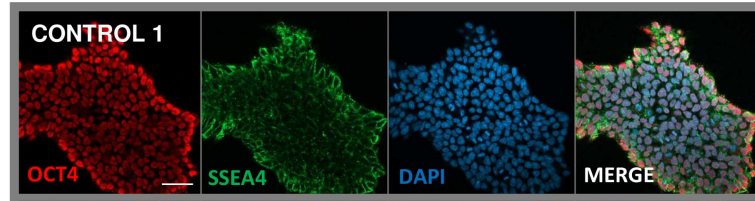

CONTROL 2
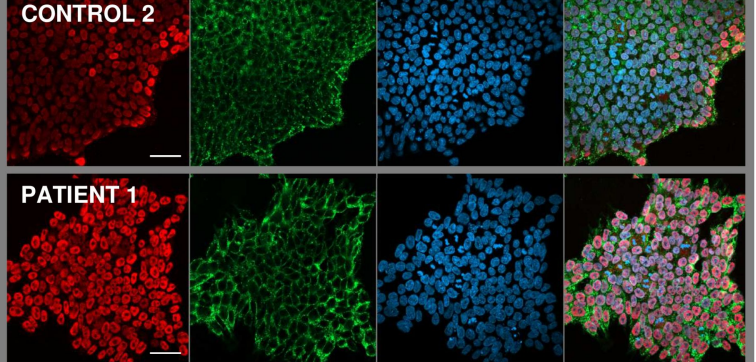

PATIENT 2

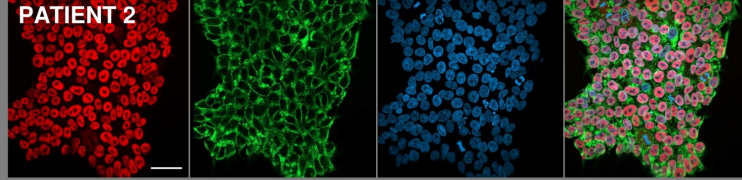

PATIENT 3 
A
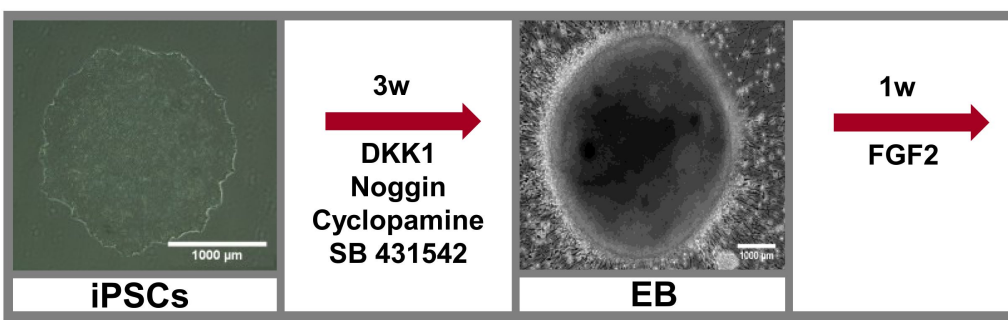

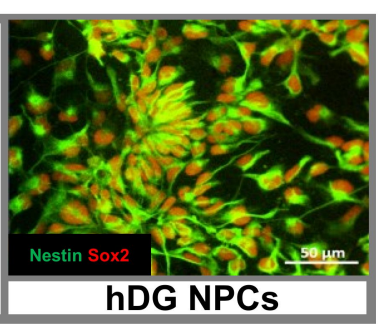

hDG NPCs

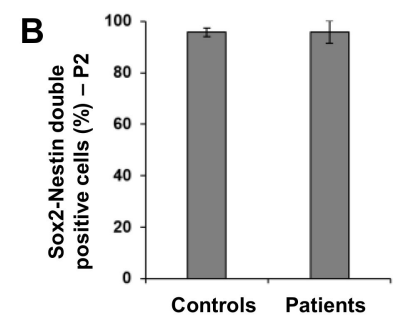

C

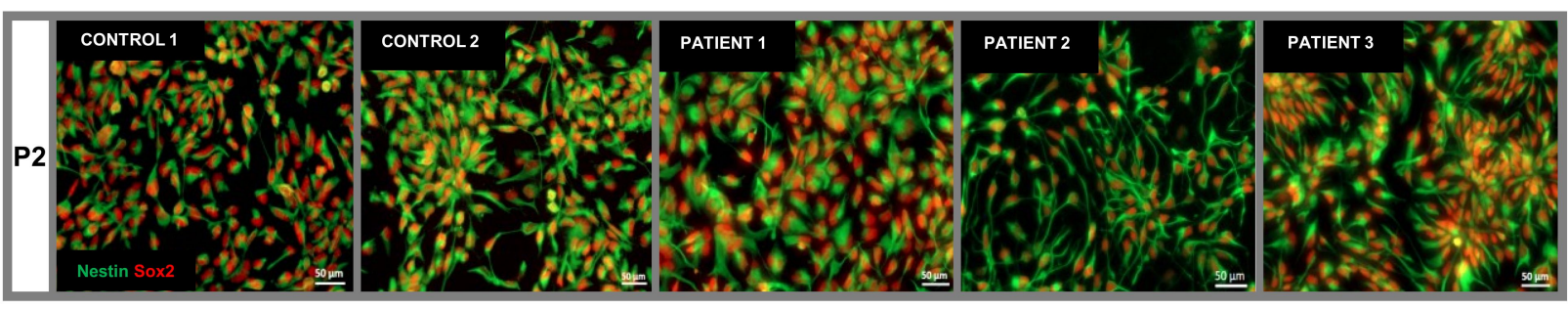

D

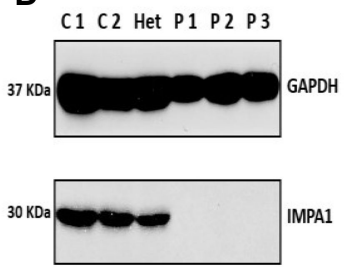

\section{E 1,057 upregulated genes}

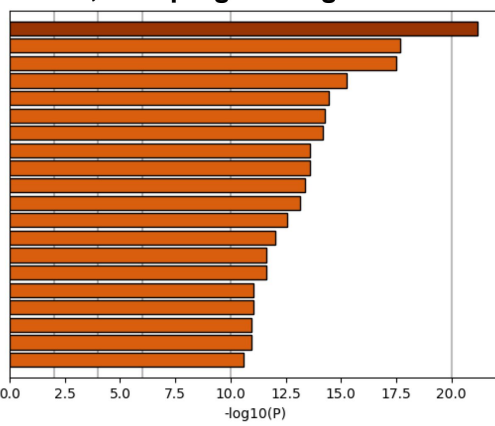

\section{F 633 downregulated genes}

GO:0043062: extracellular structure organization GO:0003002: regionalization

GO:0007423: sensory organ development 0.0007156 : homophilic cell adhesion via plasma membrane adhesion molecules GO:0001501: skeletal system development Go:0048729: tissue morphogenesis GO:0021871: forebrain regionalization GO:0070848: response to growth facto GO:0042476: odontogenesis GO:0060541: respiratory system development GO:0060485: mesenchyme development GO:0001568: blood vessel development GO:0042063: gliogenesis

GO:0010469: regultion of signaling receptor activity GO:0021536: diencephalon development GO:0007610: behavio

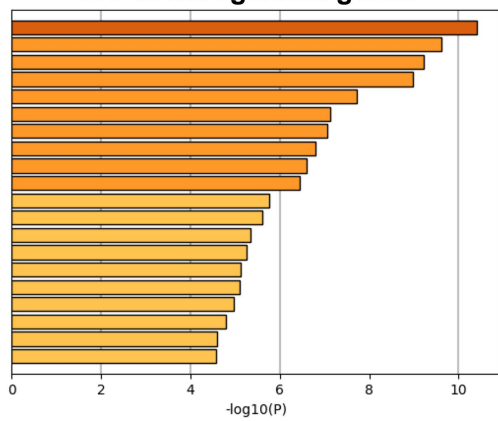

GO:0045165: cell fate commitment GO:0003002: regionalization GO:0048705: skeletal system morphogenesis GO:0007423: sensory organ development .0.0035282: segmentation O.0045057 regulation of neuron differentiation GO:0016103: diterpenoid catabolic pross GO:0030902: hindbrain development GO:0071363: cellular response to growth factor stimulus GO:0045596: negative regulation of GO:0007610: behavior G0:0007517: muscle organ development GO:0043583: ear development 0:0050878: regulation of body fluid levels GO:0021603: cranial nerve formation GO:0030326: embryonic limb morphogenesis
GO:0032526: response to retinoic acid

G

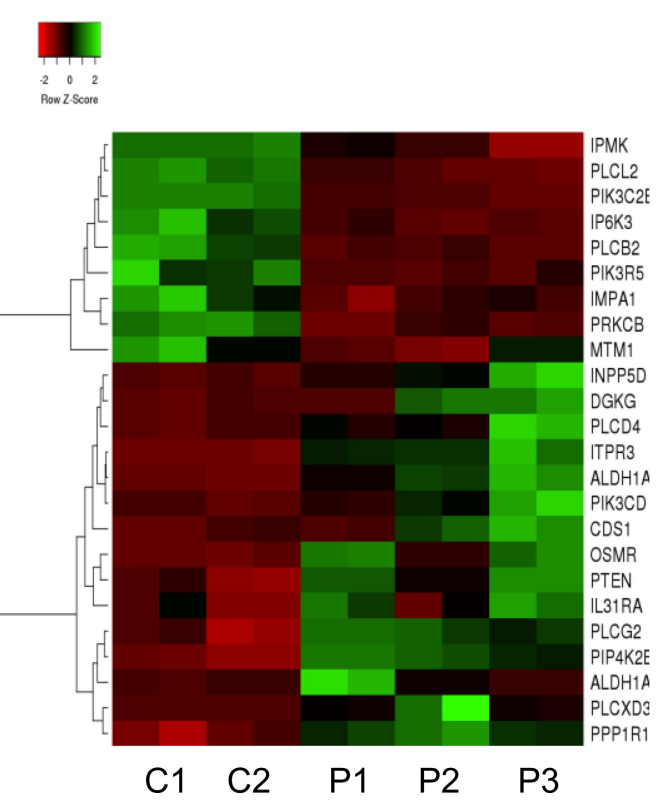

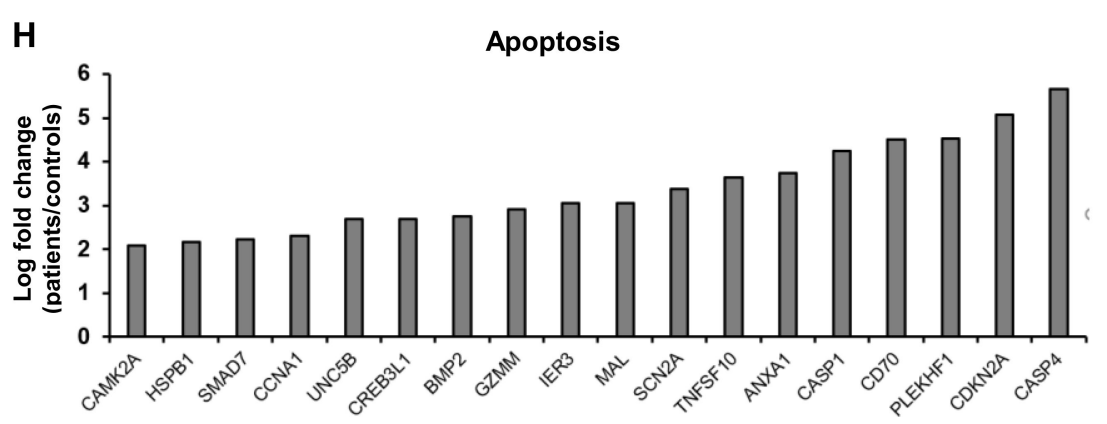

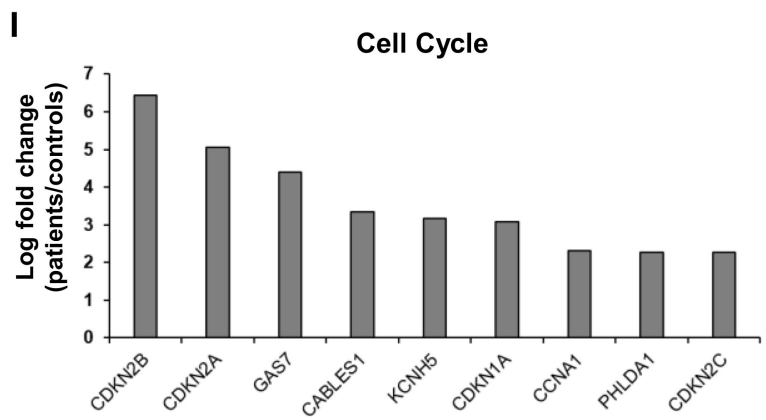



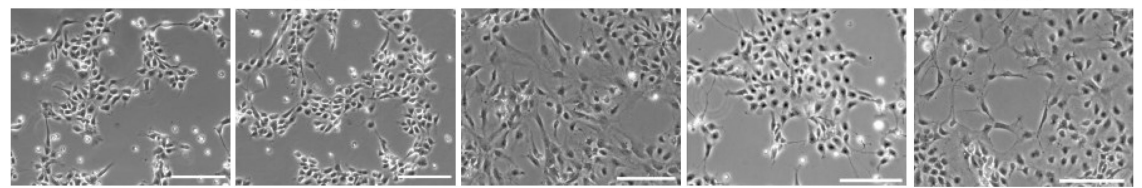

B

Control 1

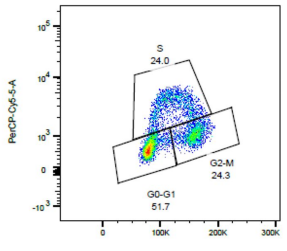

C

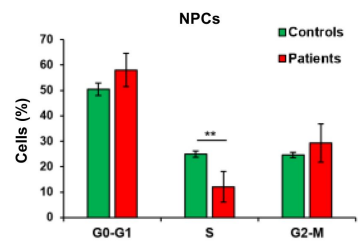

Control 2

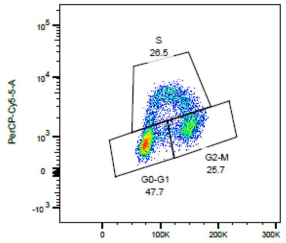

BV421-A
Patient 2

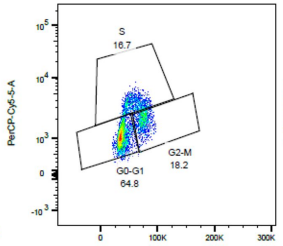

Cell Cycle

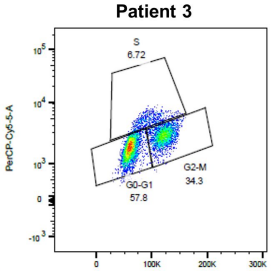

BV421-A
iPSCs

GPCs
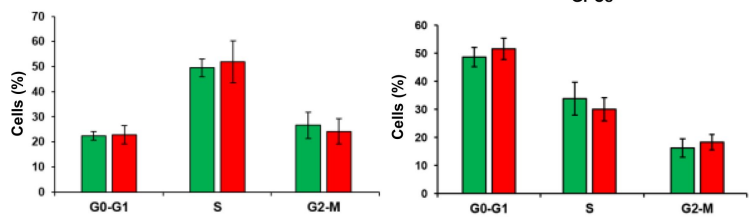

D

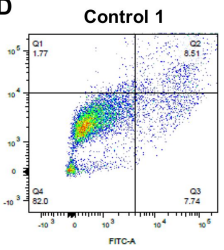

E

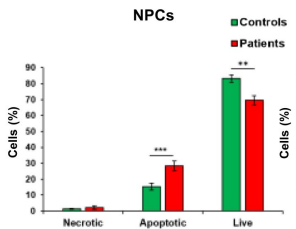

Control 2

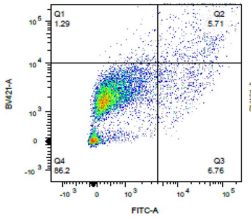

FrTCA

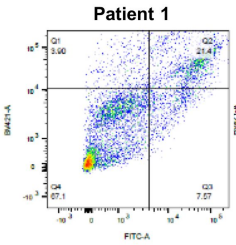

Apoptosis
Neurons (3d)

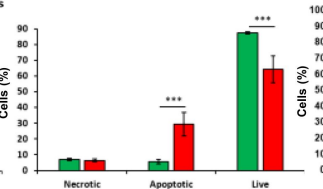

Patient 2

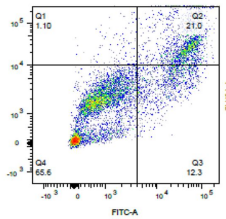

iPSCs
Patient 3

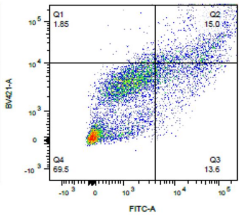

GPCs
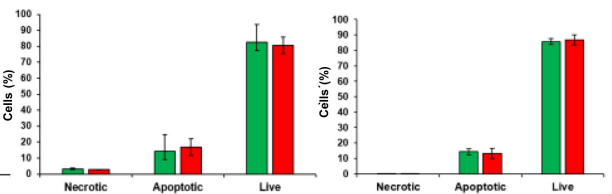

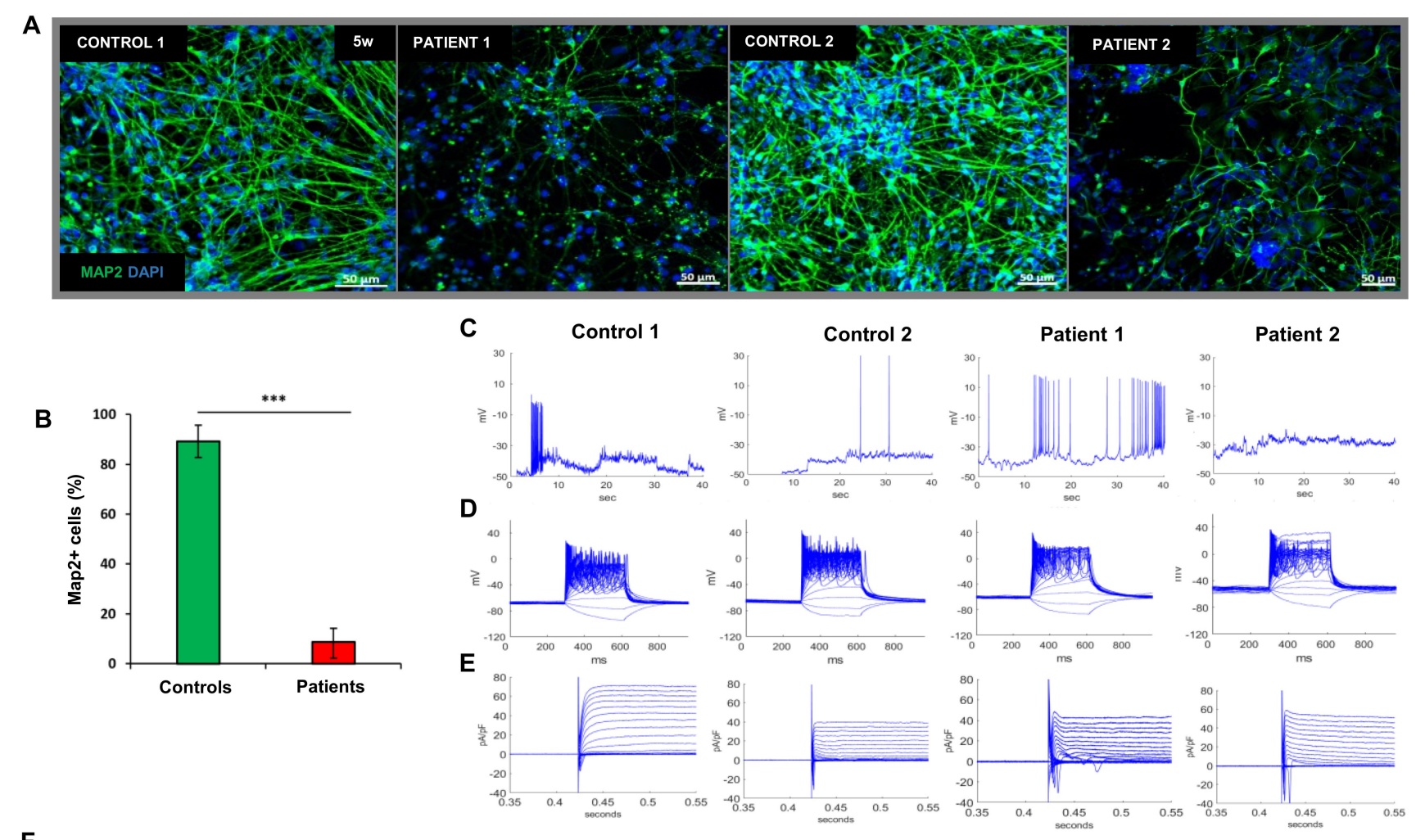

$\mathbf{F}$

685 downregulated genes

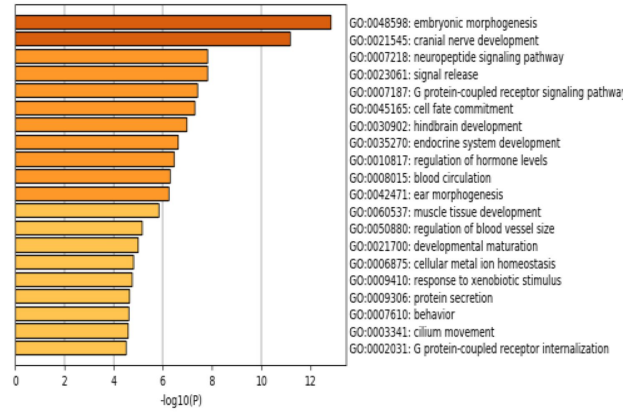

G
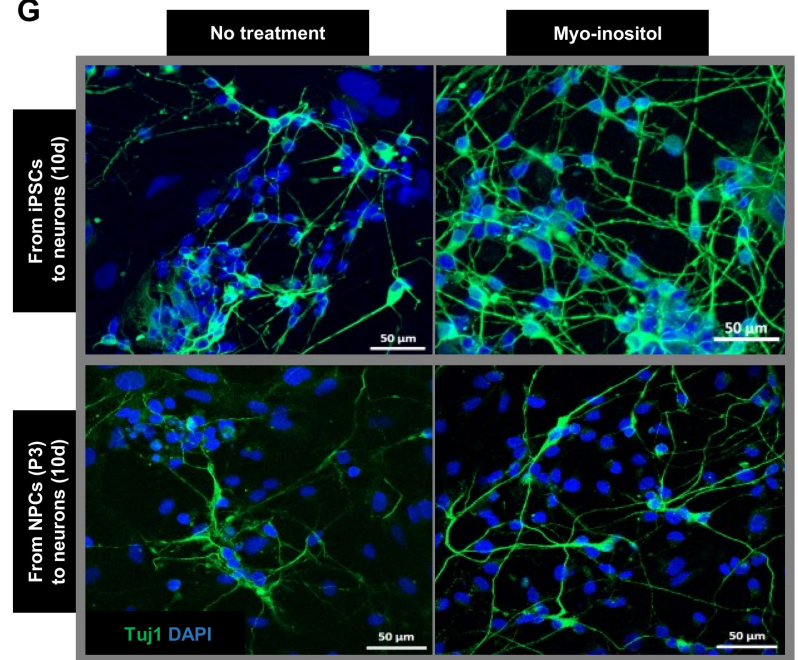

661 upregulated genes

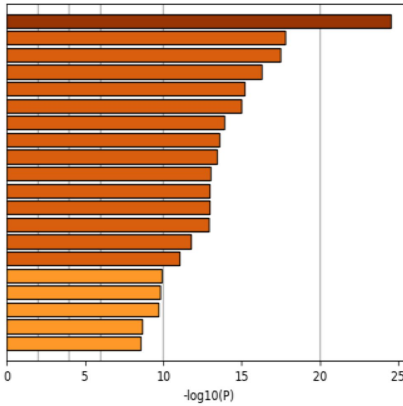

G0:0030855: epithelial cell differentiation 60:0030198: extracellular matrix organization G0:0003002: regionalization G0:0021871: forebrain regionalization G0:0048729: tissue morphogenesis 0:0048608: reproductive structure development 0:0007423: sensory organ development 60:0001655: urogenital system development G0:0042476: : odontogenesis G0:0060541: respiratory system development GO:0048598: embryonic morphogenesis G0:0001568: blood vessel development G0:0042063: gliogenesis

to growth factor . GO:0060425: lung morphogenesis

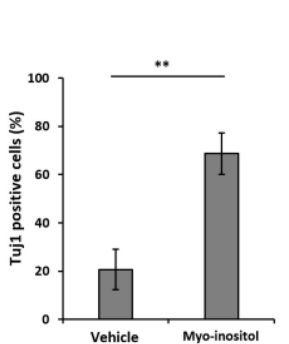

H
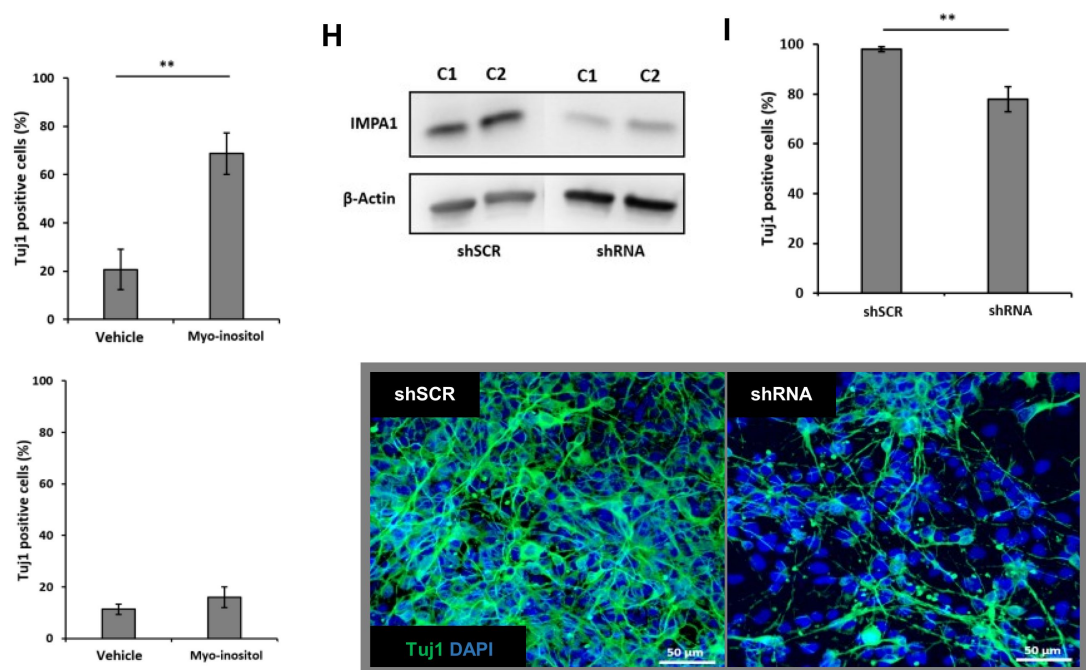

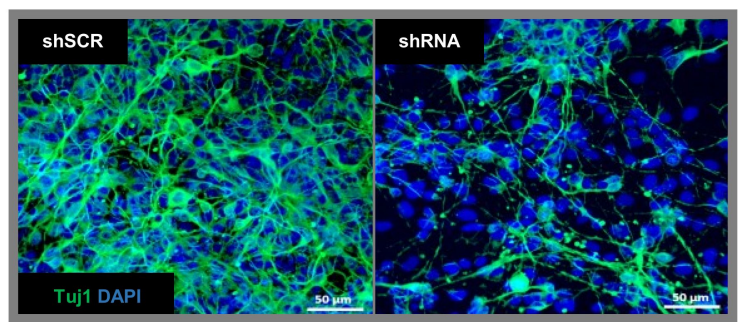


A

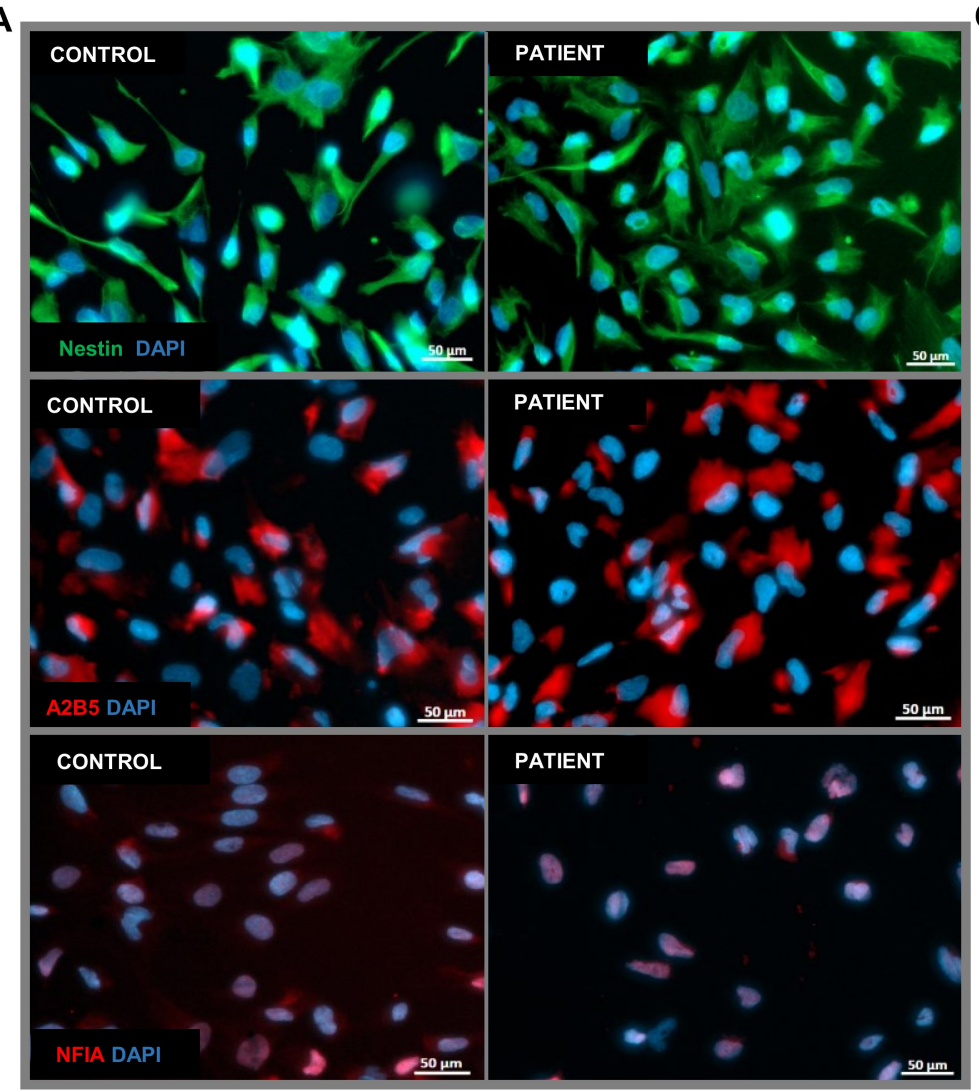

B

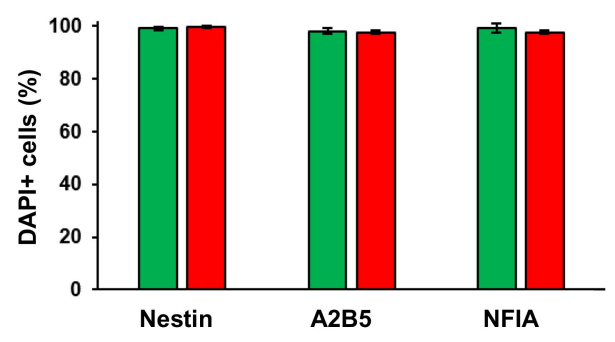

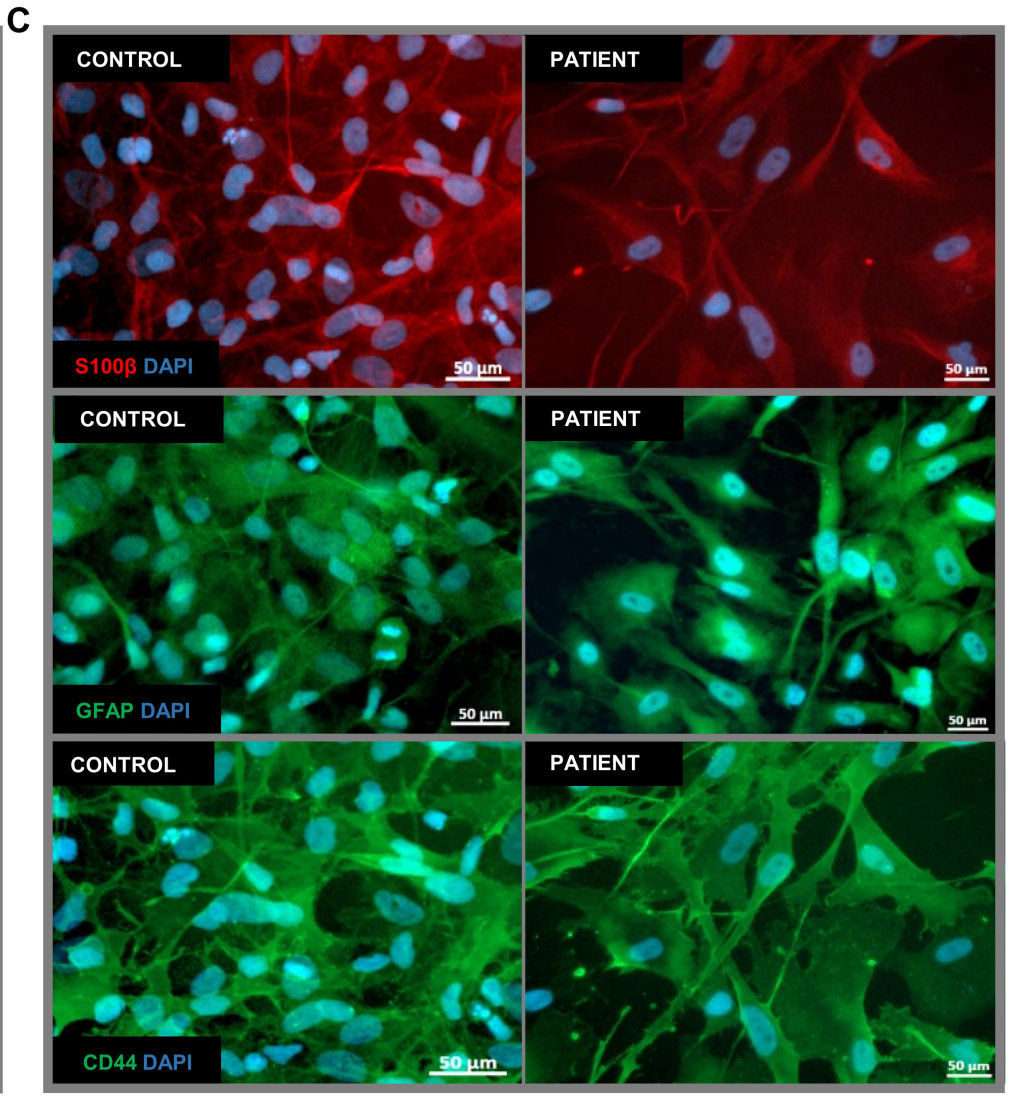

D

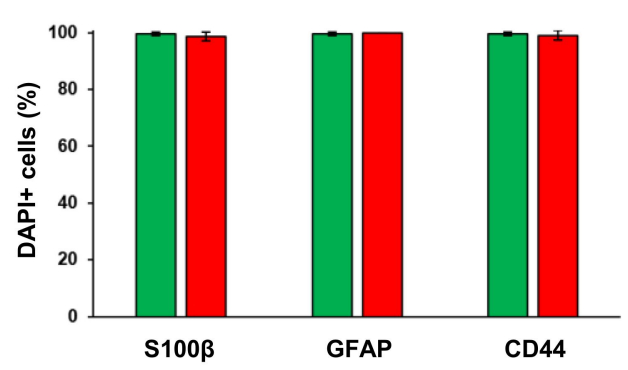

E

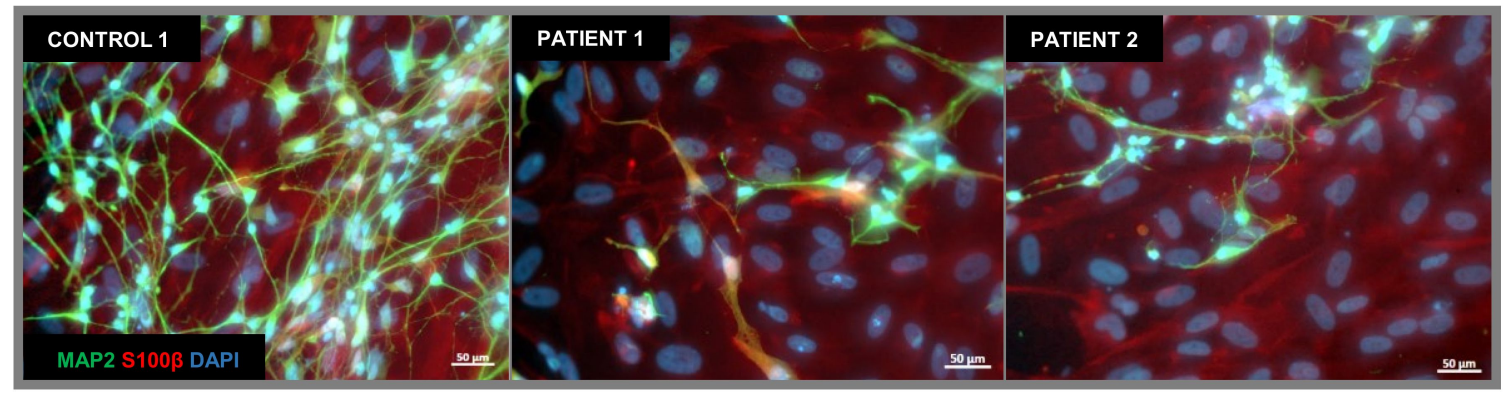

